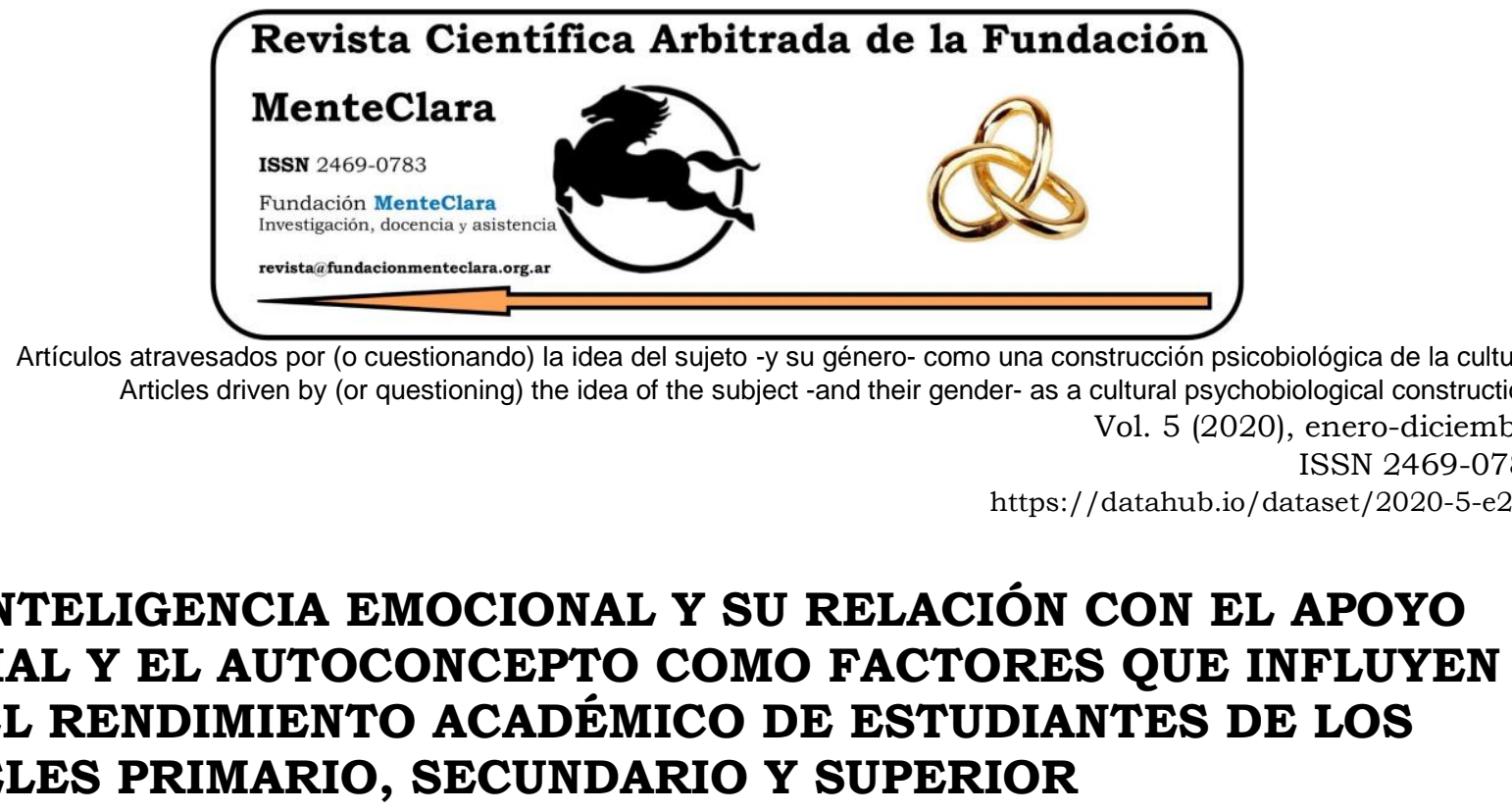

\title{
LA INTELIGENCIA EMOCIONAL Y SU RELACIÓN CON EL APOYO SOCIAL Y EL AUTOCONCEPTO COMO FACTORES QUE INFLUYEN EN EL RENDIMIENTO ACADÉMICO DE ESTUDIANTES DE LOS NIVELES PRIMARIO, SECUNDARIO Y SUPERIOR
}

EMOTIONAL INTELLIGENCE AND ITS RELATIONSHIP TO SOCIAL SUPPORT AND SELF- CONCEPT AS FACTORS THAT INFLUENCE THE ACADEMIC PERFORMANCE OF STUDENTS AT THE PRIMARY, SECONDARY AND HIGHER LEVELS

Patricia Medina patricia.medina@uflouniversidad.edu.ar

Facultad de Psicología y Ciencias Sociales, Universidad de Flores, Argentina

Cómo citar este artículo / Citation: Medina, P. (2020). La inteligencia emocional y su relación con el apoyo social y el autoconcepto como factores que influyen en el rendimiento académico de estudiantes de los niveles primario, secundario y superior. Revista Científica Arbitrada de la Fundación MenteClara, Vol.5 (203). DOI:

https://doi.org/10.32351/rca.v5.203

Copyright: (C) 2020 RCAFMC. Este artículo de acceso abierto es distribuido bajo los términos de la licencia Creative Commons Attribution 4.0 International License (CC BY 4.0). Recibido: 29/11/2020. Aceptado: 08/12/2020 Publicación online: 10/12/2020

Conflicto de intereses: Ninguno que declarar.

\section{Resumen}

El ámbito escolar constituye un espacio en que los estudiantes deben sortear múltiples retos en su acercamiento al proceso de aprendizaje. Se ha demostrado que los componentes emocionales cumplen un rol fundante en el desempeño académico escolar, siendo este el eje de interés que motiva el presente trabajo. Partiendo de una revisión bibliográfica por diversas revistas académicas se propone indagar en qué medida la inteligencia emocional en conjunción con el apoyo social y el autoconcepto influyen en el desempeño académico de estudiantes de distintos niveles del sistema educativo. Con respecto a la Inteligencia emocional se arriba a resultados divergentes, hecho que pudiera relacionarse con la multiplicidad de instrumentos con los que la variable ha sido evaluada. Al correlacionar Inteligencia Emocional con Apoyo Social y Autoconcepto se advierte que suelen tener una influencia significativa en el buen 
desempeño educativo de los estudiantes de los niveles primarios, secundario y superior.

\section{Abstract}

The educational environment constitutes multiply challenges to approach learning. It has been demonstrated that the emotional components take an important part in the educational process, being this an important issue that motivates this project. Starting from a bibliographic review from various academic magazines it is proposed to investigate in which extend the emotional intelligence in conjunction with the social support and the self-concept influences in the academic performance of the students in the different levels of the educational system

As regards the emotional intelligence we reach divergent results, as a result of a variety of instruments used to evaluate it. When we correlate emotional intelligence with social support and self -concept we realize that it has a significant influence in the educational performance of primary, secondary, and high-level students.

Palabras Claves: Inteligencia Emocional; Rendimiento académico; Apoyo social; Autoconcepto

Keywords: emotional intelligence; academic performance; social support; self-concept 


\section{Introducción}

El acto de aprendizaje sistemático y acreditación de niveles educativos supone, en ocasiones, la superación de diversos obstáculos por parte de estudiantes. Aquí, los componentes emocionales juegan un rol fundante.

Diversos estudios (Morales \& López Zafra, 2009), (Castaño Castrillón \& Pérez Cala, 2014), (Buenrostro Guerrero et. al, 2012) se ocupan de la influencia de la inteligencia emocional en el rendimiento educativo. Dando continuidad a dichos estudios, se propone indagar el rol de la inteligencia emocional en el tránsito educativo, correlacionando el papel que juegan el apoyo social y el autoconcepto con esta primera variable.

\section{Inteligencia emocional}

Goleman (1997) conceptualiza la inteligencia emocional a partir de cinco principios que le permiten entender de un modo novedoso los procesos cognitivos. Autoconciencia emocional, autorregulación emocional, automotivación, empatía y administración de las relaciones, constituyen los elementos con los cuales supera la mirada lógica y racional, que, tradicionalmente, circundó el concepto de inteligencia.

La inteligencia emocional supone la capacidad de un individuo para comprender, controlar y trabajar sus propias emociones y reconocer las ajenas, en pos de conseguir resultados positivos en su desempeño social, laboral, interrelacional. (Salovey \& Mayer, 1990) Estos autores acuñan este concepto estableciendo una jerarquización de habilidades que se inician con la percepción emocional arribando a la regulación emocional como el estado de mayor complejidad. 


\section{Inteligencia emocional y rendimiento académico}

Las personas que desarrollan su inteligencia emocional son capaces de encausar las emociones negativas a fin de que no obstaculicen la resolución de las distintas situaciones que se le presentan en el cotidiano. La escuela constituye un ámbito donde los individuos son enfrentados a diversos retos en que deben poner en práctica sus capacidades personales, -intelectuales, sociales, emocionales-. Por lo antedicho, se propone indagar la influencia de la inteligencia emocional en el rendimiento académico.

Valenzuela Santoyo y Portillo Peñuelas (2016) realizan un estudio sobre la incidencia de la inteligencia emocional en el rendimiento escolar de 58 alumnos de $5^{\circ}$ y $6^{\circ}$ año del nivel primario de Sonora, México. Siguiendo la propuesta de Goleman (1997) definen la inteligencia emocional como la capacidad de reconocer sentimientos propios y ajenos y manejarlos. En este sentido, los autores conceptualizan la inteligencia emocional como las habilidades personales, afectivas y sociales que ayudan a una persona a conocerse a sí misma y a relacionarse mejor con los demás. Valenzuela y Portillo (2018) definen el rendimiento académico como la productividad del sujeto, remitiendo a las percepciones, rasgos y actividades que, por aplicación del propio esfuerzo, generan un producto final. En su análisis, estiman que, en la medida que alumnos comprendan las causas, escenarios y circunstancias que promueven la generación de múltiples sentimientos, serán capaces de manejar sus emociones. A partir de dichas capacidades podrán contar con los recursos necesarios para modificar su pensamiento y buscar así alternativas de solución a sus problemas, comportamientos estos, que potenciarán un buen desempeño escolar. 
Chuquimantari (2017) realiza un estudio sobre la correlación existente entre Inteligencia emocional y rendimiento académico en estudiantes de la Universidad Continental, Huancayo. Construye una muestra censal de 246 alumnos de primer semestre de la carrera de Psicología a quienes administra una encuesta y un cuestionario. Del análisis de las variables, no se arriba a resultados que pueden comprobar la relación intrínseca entre estos aspectos, concluyendo que no existe relación directa ni significativa entre las variables inteligencia emocional y rendimiento académico en los estudiantes de la carrera profesional de Psicología de la Universidad Continental.

Chambi y Pérez (2018) presentan un estudio correlacional entre inteligencia emocional y rendimiento académico de 235 estudiantes, de tercero a sexto grado, de una escuela confesional de nivel primario de la selva peruana. En dicho estudio aplican el Inventario de Inteligencia Emocional de Barón ICE NA, y valoran las calificaciones de las áreas de las áreas de Comunicación y Matemática como instrumentos que den cuenta del rendimiento académico escolar. Como resultado encuentran, al igual que Chuquimantari (2017), que no existe relación significativa entre la inteligencia emocional y el rendimiento académico en el área de curricular de comunicación. Sin embargo, si se presenta una influencia de la inteligencia emocional en el rendimiento académico en el área de matemáticas, hecho que permite hipotetizar que el aprendizaje de las ciencias denominadas "duras" podria ser favorecido por ciertas competencias emocionales.

Acosta y Clavero (2017) se proponen conocer los predictores de la Inteligencia Emocional y el Rendimiento Académico de 404 de alumnos, de 6 a 12 años, en la ciudad de Ceuta. Como instrumento de evaluación emplean una adaptación propia del MSCEIT "Test de Inteligencia Emocional de Mayer, Salovey \& Caruso" y las calificaciones de los 
alumnos. Los resultados arriban a una relación directamente proporcional entre la inteligencia emocional y el rendimiento escolar, hecho que demuestra que las habilidades emocionales tienen una influencia evidente en el logro académico. Los autores concluyen exponiendo la importancia de promover las competencias emocionales como medida necesaria para mejorar el proceso de aprendizaje del alumnado.

\section{Inteligencia emocional y apoyo social}

El apoyo social es el conjunto de provisiones instrumentales que las redes sociales, comunitarias y afectivas asignan a una persona. E1 vínculo de ésta con el entorno se representa en tres niveles: la comunidad, las redes sociales y relaciones íntimas y de confianza. (Lin, 1986)

Puesto que los resultados sobre la influencia de la inteligencia emocional en el rendimiento escolar resultan divergentes, se propone conocer si la variable apoyo social en consonancia con la inteligencia emocional influyen en el rendimiento académico.

Fernández-Lasarte, et al. (2019) estudian la correlación entre Rendimiento académico, apoyo social percibido e inteligencia emocional en 419 estudiantes de la Universidad del País Vasco con edades entre 18 y 40 años. Los resultados obtenidos por esta investigación apoyan la existencia de relaciones positivas y significativas entre el apoyo social percibido, la Inteligencia emocional y el rendimiento académico. En la investigación se muestra que el apoyo de la familia y el apoyo de las amistades son proveedoras de apoyo emocional, por ello, tanto la familia como las amistades podrian ser favorecedoras de un mayor desarrollo de la Inteligencia emocional. Los hallazgos expresan que, entre la población 
universitaria, la familia continúa siendo la principal fuente de vinculación emocional por lo que ha de ofrecer apoyo para la mejora de la Inteligencia Emocional en las dimensiones de comprensión y regulación adecuada de las emociones. Concluyen demostrando las elevadas asociaciones entre el apoyo docente con el rendimiento académico y el apoyo familiar con la reparación emocional y la reparación emocional, componentes estos de la inteligencia emocional con efectos sobre el rendimiento académico.

Agirre, et al. (2019) presentan un estudio en que examinan la influencia de las principales fuentes de apoyo social y de la inteligencia emocional sobre el rendimiento académico de 850 estudiantes del nivel secundario del País Vasco. Los datos obtenidos con regresiones múltiples evidencian que el apoyo que el alumnado percibe tener de su familia y docentes tiene un efecto directo y relevante sobre el logro académico en la Educación Secundaria. Asimismo, el trabajo destaca la importancia del apoyo del grupo familiar y de los docentes en el ajuste sociopersonal por sobre la inteligencia emocional percibida en el desarrollo educativo. Según este estudio, la Inteligencia emocional no tendría una relación directa con el rendimiento escolar. Para los autores, la única dimensión de la Inteligencia emocional que parece incidir en el rendimiento académico es la atención emocional, siendo su efecto negativo y limitándose dicha influencia al conjunto de estudiantes femeninas. Concluyen indicando que prestar demasiada atención a las emociones podria estar contribuyendo a incrementar un pensamiento rumiativo, y, por consiguiente, a poner en riesgo su ajuste personal y escolar.

Azpiazu, et al. (2015) se proponen analizar la relación entre el apoyo social familiar, de amistades y docente; y las dimensiones de atención, claridad y reparación de la inteligencia emocional. Sobre una muestra de 1543 adolescentes - 728 chicos varones y el resto estudiantes mujeres de 
escuelas secundarias del País Vasco, evalúan la capacidad predictiva del apoyo social en la inteligencia emocional de los estudiantes adolescentes. Los resultados del estudio señalan que el apoyo social y la inteligencia emocional se encuentran significativamente relacionados tanto para la muestra masculina como femenina. En el caso de los varones, el apoyo de las amistades predice una mayor atención emocional y claridad en los sentimientos, mientras que el apoyo familiar estimula una mayor regulación emocional. En cuanto a las alumnas, el apoyo de los pares promueve la atención emocional, y el apoyo familiar, la claridad y regulación emocional. Estos hallazgos confirman la influencia de los pares y la familia en el desarrollo adolescente.

A partir de la lectura realizada, es factible arribar a la conclusión de que el apoyo social se constituye en un recurso favorecedor para el desarrollo de la inteligencia emocional, siendo esta una capacidad necesaria para operar con certeza en las relaciones personales y funciones productivas, entre ellas, las escolares.

\section{Inteligencia emocional y autoconcepto}

$\mathrm{El}$ autoconcepto es definido como las percepciones que los individuos construyen de sí mismo basándose en sus propias experiencias y en las valoraciones que reciben de los demás respecto a sus conductas. El autoconcepto remite a la concepción que cada uno construye respecto a su ser físico, social y espiritual (Callaza Luna \& Molero, 2013). Puesto que el autoconcepto resulta un eje central del desarrollo de la personalidad, es relevante conocer sus efectos a nivel educativo.

Carrillo Ramírez, et al. (2020) estudian la inteligencia emocional -en sus factores de atención, claridad y reparación emocional considerándola un predictor del autoconcepto. Consideran, además, que 
un adecuado autoconcepto contribuye en la calidad de las relaciones interpersonales, mejorando con ello el ajuste social de los individuos. Los resultados de su estudio demuestran que la inteligencia emocional contribuye positiva y significativamente en la construcción del autoconcepto. La relación positiva entre inteligencia emocional percibida y autoconcepto resulta relevante dentro del ámbito educativo. De desarrollarse estrategias que promuevan la educación emocional entre los jóvenes se respondería a necesidades socio afectivas que no suelen incluirse en los programas de educación formal pero que, indudablemente, tienen consecuencias en el éxito o fracaso académico de los estudiantes.

Agirre, et al. (2015) estudian la capacidad predictiva del autoconcepto multidimensional -físico, social, personal, académico y general- y las dimensiones de atención, claridad y reparación de la inteligencia emocional en el ajuste escolar percibido en una población de 1.543 adolescentes de escuelas públicas y privadas de Educación Media de la Comunidad Autónoma Vasca. En el caso de los alumnos varones, llegan a la conclusión de que la reparación emocional, autoconcepto académico, autoconcepto social y autoconcepto general predicen significativamente el ajuste escolar. En el caso de las adolescentes mujeres encuentran que, únicamente, las variables académicas, personal y general del autoconcepto predicen significativamente el ajuste escolar. A partir de estos resultados, los autores expresan que el autoconcepto ejerce una influencia de mayor significación respecto al ajuste escolar que la inteligencia emocional percibida 


\section{Inteligencia emocional, apoyo social percibido, autoconcepto y rendimiento académico}

Por último, Fernández Lazarte, et al. (2019) analizan las relaciones entre el autoconcepto, el apoyo social percibido y la reparación emocional como predictores del rendimiento académico en estudiantes de nivel secundario y universitario de la Comunidad Vasca. La reparación emocional, en coincidencia con la perspectiva aquí sostenida, constituye un factor de la inteligencia emocional del modelo de Salovey y Mayer. El autoconcepto se define como la percepción que la persona tiene de si formada por sus experiencias y relaciones ambientales. El apoyo social percibido remite al rol de los profesores en el rendimiento académico de los estudiantes. Los resultados demuestran que el apoyo docente es un predictor del rendimiento académico de los estudiantes universitarios precedido por el autoconcepto. En los estudiantes de nivel secundario, el principal predictor es autoconcepto, luego el apoyo percibido y por última la reparación emocional. Los autores concluyen expresando la importancia de proponer buenas prácticas educativas como variables propias del apoyo percibido por la influencia que tendrian en el rendimiento académico y las consecuencias respecto al autoconcepto y la reparación emocional.

\section{Conclusión}

En el presente trabajo se propuso conocer la correlación de la inteligencia emocional con el rendimiento académico, el apoyo social y el autoconcepto de estudiantes de diversos niveles educativos.

Respecto a la relación de la inteligencia emocional y rendimiento académico, los hallazgos presentan resultados divergentes sobre la función de la inteligencia emocional en el desempeño educativo de los 
estudiantes. Si bien algunos estudios, Chuquimantari, J. S. (2017) y Chambi, D. P., y Pérez, S. E. R. (2018) descartan una correlación entre ambas variables, otros establecen la influencia de la inteligencia emocional como un factor predictivo del buen rendimiento académico escolar.

Ante la variabilidad de resultados, se propone indagar sobre la relación entre la inteligencia emocional y el apoyo social percibido y/o el autoconcepto. Los estudios presentados demuestran que existe una influencia altamente significativa de los apoyos sociales en la constitución de la inteligencia emocional, aspecto que resulta promotor del desempeño educativo de niños y jóvenes. El rol que asumen la red de relaciones personales, amistades y docentes constituiría un apoyo emocional con implicancias en el aprendizaje de los estudiantes.

El autoconcepto y la inteligencia emocional mantienen una correlación significativa en la educación de las poblaciones estudiadas. Los sujetos con un autoconcepto positivo y una adecuada inteligencia emocional que le permite identificar y tramitar sus emociones- suelen tener un buen rendimiento académico.

A lo largo del presente trabajo de revisión bibliográfica, se advierte que la inteligencia emocional es un constructo que ha sido evaluada con diversas escalas, inventarios y cuestionarios. La multiplicidad de instrumentos utilizados podría ser el motivo por el cual los estudios encuentran resultados disimiles. Continuar estudiando esta variable, estableciendo acuerdos respecto a su evaluación, constituye una necesidad del campo científico.

Por todo lo expuesto, se considera que el abordaje de la inteligencia emocional entendida como la capacidad de monitorizar los sentimientos y emociones propios y de los demás, de discriminar entre ellos y utilizar 
esta información para guiar el pensamiento de uno y acciones (FragosoLuzuriaga, 2015), constituye una politica fundamental a nivel educativo por sus efectos positivos en el rendimiento académico de los estudiantes, acción que redunda en el autoconcepto de los estudiantes y reconoce el valor del apoyo percibido- por sus referentes cercanos- en la educación de los individuos. 


\section{Referencias}

Acosta, F. P., \& Clavero, F. H. (2017). La inteligencia emocional como predictora del rendimiento académico en la infancia: el contexto pluricultural de Ceuta. Revista Complutense de Educación, 28(4), 1251. https://dx.doi.org/10.5209/RCED

Agirre, I. A., Izaguirre, L. A., Etxaniz, I. E., \& Maya, M. S. (2015). Capacidad predictiva del autoconcepto y la inteligencia emocional en el ajuste escolar autopercibido. Bordón. Revista de pedagogía, 67(4), 9-26. https://doi.org/10.13042/Bordon.2015.67401

Antonio-Agirre, I., Rodríguez-Fernández, A., \& Revuelta, L. (2019). El impacto del apoyo social y la inteligencia emocional percibida sobre el rendimiento académico en Educación Secundaria. European Journal of Investigation in Health, Psychology and Education, 9(2), 109-118. https://doi.org/10.30552/ejihpe.v9i2.324

Azpiazu, L., Esnaola, I., \& Sarasa, M. (2015). Capacidad predictiva del apoyo social en la inteligencia emocional de adolescentes. European journal of education and psychology, 8(1), 23-29. https://doi.org/10.1016/j.ejeps.2015.10.003

Buenrostro-Guerrero, A. E., Valadez-Sierra, M. D., Soltero-Avelar, R.., Nava-Bustos, G.., Zambrano-Guzmán, R.., \& García-Garcia, A. (2012). Inteligencia emocional y rendimiento académico en adolescentes. Revista de educación y Desarrollo, 20(1), 29-37

Carrillo-Ramírez, E, Pérez-Verduzco, G., Laca-Arocena, F. A., \& Luna-Bernal, A. C. A. (2020). Inteligencia emocional percibida y autoconcepto en adolescentes estudiantes de bachillerato. Contenido/Summary, 14(4), 33.

Castaño Castrillón, J. J., \& Páez Cala, M. L. (2014). Inteligencia emocional y rendimiento académico en estudiantes universitarios. Psicología desde el Caribe, 32(2), 268-285. http://dx.doi.org/10.14482/psdc.32.2.5798

Chambi, D. P., \& Pérez, S. E. R. (2018). Inteligencia emocional y rendimiento académico en estudiantes de nivel primario en una institución educativa confesional de la selva peruana. Revista Muro de la Investigación, 3(1), 30-39.

https://doi.org/10.17162/rmi.v3i1.1112

Chuquimantari, J. S. (2017). Inteligencia emocional y rendimiento académico en estudiantes de la Universidad Continental, Huancayo. Apuntes de Ciencia \& Sociedad, 7(2), 125-132. https://doi.org/10.18259/acs.2017017

Fernández Lasarte, O., Díaz, E. R., \& Sáez, I. A. (2019). Rendimiento académico, apoyo social percibido e inteligencia emocional en la universidad. EJIHPE: European Journal of Investigation in Health, Psychology and Education, 9(1), 39-49. doi:

https://doi.org/10.30552/ejihpe.v9i1.315 
Fernández Lazarte, O Ramos Diaz, E, Goñi, E \& Rodríguez Fernández, A (2019). Estudio comparativo entre educación superior y educación secundaria: efecto del apoyo social percibido, el autoconcepto y la reparación emocional en el rendimiento académico. Educación XXI. 22 (2) 165-185. https://doi.org/10.5944/educxx1.22.2

Fragoso-Luzuriaga, R. (2015). Inteligencia y competencias emocionales en educación superior ¿un mismo concepto? Revista iberoamericana de educación superior, 6(16), 110-125.

Goleman, D. (1997). La inteligencia emocional, Porqué es más importante que el coeficiente intelectual. Kairos

Lin, N. (1986). Conceptualising social support. En Lin, N, Dean, A \& En T. Sel, Social support, life events and depression. Academics Press

Luna, N. C., \& Molero, D. (2013). Revisión teórica sobre el autoconcepto y su importancia en la adolescencia. Revista electrónica de investigación y docencia (REID), (10), 43-64

Morales, M. I. J., \& López-Zafra, E. (2009). Inteligencia emocional y rendimiento escolar: estado actual de la cuestión. Revista Latinoamericana de psicología, 41(1), 69-79.

Reina, I. (2009). La inteligencia emocional en el proceso de enseñanza-aprendizaje: concepto y componentes. Revista Innovación y experiencias educativas, (14), 1-12.

Salovey, P., \& Mayer, J. (1990). Inteligencia emocional. Imaginación, conocimiento y personalidad, 9(3), 185-211.

Valenzuela-Santoyo, A. D. C., \& Portillo-Peñuelas, S. A. (2018). La inteligencia emocional en educación primaria y su relación con el rendimiento académico. Revista Electrónica Educare, 22(3), 228-242. http://dx.doi.org/10.15359/ree.22-3.11 